\title{
Real-world outcomes of stem cell transplantation for severe aplastic anemia: A single-center experience in Northern India
}

\author{
Dharma Choudhury ${ }^{1}$, Meet Kumar ${ }^{1}$, Sanjeev Sharma ${ }^{1}$, Vipin Khandelwal ${ }^{1}$, Divya Doval $^{1}$, Tina Dadu $^{2}$, Rasika Setia ${ }^{3}$, \\ Anil Handoo ${ }^{2}$
}

${ }^{1}$ Department of Hematology and BMT, BLK Super Specialty Hospital, New Delhi, ${ }^{2}$ Department of Hematology, BLK Super Specialty Hospital, New Delhi, ${ }^{3}$ Department of Transfusion Medicine, Super Specialty Hospital, New Delhi

\section{Abstract}

Introduction: Stem cell transplantation is the cornerstone of therapy for transplant-eligible patients with severe aplastic anemia.

Materials and methods: Patients with severe aplastic anemia undergoing stem cell transplantation (including matched haplo-identical related donors) with a standard conditioning regimen and graft-versus-host disease (GVHD) prophylaxis were analyzed. High-risk patients were identified as having undergone $>20$ pre-transplant transfusions, having febrile neutropenia at the time of transplantation, or having undergone failed immunosuppressive therapy.

Results: A total of 111 patients underwent stem cell transplantation, with a median age of 17 years. Seventy-six patients received matched related donor (MRD) transplants, and 35 received haplo-identical donor (HID) transplants. Among all patients, $65.7 \%$ were high-risk patients, with a significantly higher proportion among those receiving HID transplants (38\% for MRD vs. $83 \%$ for HID). Acute GVHD grades $2-4$ was observed in $9 \%$ of patients, and chronic GVHD in $16.2 \%$ of patients. Primary graft rejection was more common in $9.9 \%$ of patients (21\% for HID, 5\% for MRD). The 2-year overall survival and disease-free survival were $67 \%$ and $66 \%$, respectively, with better outcomes for MRD and low-risk HID transplants than for high-risk HID transplants. The most common cause of mortality was sepsis-related death (accounting for $27 \%$ of the total deaths). Sepsis-related early deaths were significantly more common among high-risk patients who received HID transplants.

Conclusion: We conclude that MRDs remain the preferred donor source for allogeneic stem cell transplants in patients with aplastic anemia; however, HIDs can be considered as a life-saving treatment for patients with aplastic anemia.

Key words: aplastic anemia; stem cell transplant; real-world data

Submitted December 16, 2019; Accepted January 31, 2020; Published online May 25, 2020; Issued online May 25, 2020

Correspondence: Meet Kumar, Department of Hematology and BMT, BLK Super Specialty Hospital, Pusa Road, New Delhi-11005, India, E-mail: kr_meet@yahoo.com

\section{Introduction}

Allogeneic (Allo) stem cell transplantation (SCT) and immune suppressive therapy (IST) have remained the cornerstones of therapy for patients with severe aplastic anemia (SAA) for the last four decades, with IST being considered for patients without a matched donor. However, because of non-availability of matched donors for the majority of patients and with improvements in transplant outcomes, recent studies have favored the use of transplant-based strategies for all patients over non-transplant IST-based strategies ${ }^{1}$. We retrospectively evaluated real-world data from a single center for allo-SCT in patients with SAA.

\section{Materials and Methods}

All patients with SAA diagnosed between April 2013 and October 2019 were evaluated. Patients with inherited marrow failure syndromes were excluded. The hospital 
ethics committee and institutional review board approved the study.

The preferred stem cell source was granulocyte-colony-stimulating factor (GCSF) -primed peripheral blood. Bone marrow was used for younger donors and for those who had been administered an inadequate dose of GCSFprimed peripheral blood stem cell. The target cluster of differentiation $(\mathrm{CD}) 34$ cell dose was $>2 \times 10^{6} / \mathrm{kg}$. The conditioning regimen and graft-versus-host disease (GVHD) prophylaxis were as follows: For matched related donor (MRD) transplantation, patients received fludarabine and cyclophosphamide, with or without horse anti-thymocyte globulin (ATG) as the conditioning regi$m^{2}{ }^{2}$. Standard-dose cyclosporine with methotrexate was used as GVHD prophylaxis. For haplo-identical donor (HID) transplantation, the conditioning regimen included fludarabine, cyclophosphamide, and total-body irradiation $^{3}$. GVHD prophylaxis comprised post-transplant cyclophosphamide (PTCY), tacrolimus, and methotrexate.

High-risk patients were identified as those with febrile neutropenia or documented infection (bacterial or fungal) at the time of hematopoietic stem cell transplantation (HSCT), those in whom previous IST had failed, or those who had received more than 20 red cell transfusions before $\mathrm{HSCT}^{2}$. Statistical calculations for comparisons between MRD and HID were performed using the chi square test or Student's t test, as required. Survival analysis was conducted by Kaplan-Meier curve analysis using MedCalc version 2.0.

\section{Results}

A total of 111 patients (76 men and 35 women) underwent allo-SCT for SAA, comprising 42 patients aged $<$ 18 years and 69 patients aged $>18$ years. Seventy-six patients underwent MRD transplantation, and 35 underwent HID transplantation. Overall, $65.7 \%$ of patients were high-risk, identified as having undergone $>20$ $(58.5 \%)$ pre-transplant transfusions, having febrile neutropenia at the time of transplantation $(31.5 \%)$, and having undergone failed IST previously $(19.8 \%)$, with a significantly higher number of high-risk patients receiving HID transplants $(38 \%$ for MRD, $83 \%$ for HID, $P=$ $0.011)$. The stem cell sources were GCSF-primed peripheral blood (89 patients), bone marrow (19 patients), or combined peripheral blood and bone marrow (2 patients). The median CD34 + cell dose administered was $6.1 \times$ $10^{6}$ cells $/ \mathrm{kg}$, which was significantly higher for HID transplants (median CD34 cell dose: $5.05 \times 10^{6}$ cells $/ \mathrm{kg}$ for MRD, $7.81 \times 10^{6}$ cells $/ \mathrm{kg}$ for HID, $P=0.000041$ ) (Table 1A). There was no significant difference between the two groups with respect to the median age at the time of transplantation, sex, and stem cell source (peripheral blood, bone marrow, or combined) (Table 1A).

The median times to neutrophil and platelet engraftment were 8 days (range 7-21 days) and 19 days (range 18-41 days), respectively. Grade 3-4 mucositis was observed in $7 \%$ of patients. Cytomegalovirus (CMV) reactivation was observed in 17 patients $(15.3 \%)$ during the first 100 days, and veno-occlusive disease was observed in only 2 patients undergoing MRD transplantation. There was no significant difference between MRD and HID with respect to time to engraftment (for neutrophils and platelets), mucositis grade, and early posttransplantation complications (veno-occlusive disease, hemorrhagic cystitis, and CMV reactivation rates) (Table 1B).

Acute GVHD of grades 2-4 was observed in 9\% of patients ( 7 patients with MRD transplants, 3 patients with HID transplants, $P=\mathrm{NS}$ ). Chronic GVHD was observed in 18 patients (21\% with MRD transplants vs. $6 \%$ with HID transplants, $P=0.041)$. Primary graft rejection was observed in 11 patients (5\% with MRD transplants vs. $21 \%$ with HID transplants, $P=0.042$ ) (Table 1A). Of the 6 patients in the HID cohort who experienced primary rejection, 3 died because of sepsis, and the remaining 3 underwent second transplantation and are alive. There was no secondary graft rejection. Our 2-year overall survival (OS) and 2-year disease-free survival (DFS) rates (for all patients) were $67 \%$ and $66 \%$, respectively. Multivariate analysis by Cox proportional hazards regression showed a significant impact of acute and chronic GVHD $(P<0.0001$ for both $)$ and donor type $(P<0.0001)$ on OS. DFS was impacted by the graft source $(P=0.0193)$ and pre-transplant risk status $(P=0.0284)$, in addition to acute and chronic GVHD $(P=0.0032, P=0.0090$, respectively $)$ and donor type $(P=0.0011)$. Engraftment kinetics, mucositis grade, veno-occlusive disease, and CMV reactivation had no impact. We sub-analyzed patients with MRD and HID transplants according to the risk status (as defined earlier) and found that patients with high-risk HID transplants had significantly worse 2-year OS compared with the others $(72 \%$ for low risk/high-risk MRD, low-risk HID vs. $32 \%$ for HID, $P=0.0003)$. Similarly, 2-year DFS was significantly worse for patients with high-risk HID transplants than for others $(71 \%$ for low risk/high-risk MRD, low-risk HID vs. $29 \%$ for HID, $P=0.0003)$ (Figure 1). Overall, the most common cause of death was sepsis-related $(27 \%)$, with significantly more sepsis-related deaths with HID transplants (14\% for MRD vs. $54 \%$ for HID, $P=0.00001)$.

\section{Discussion}

Allo-SCT with MRD is preferred over IST because of certain disadvantages associated with IST, including treatment failure, clonal evolution to myelodysplastic 
Table $1 \mathrm{~A}$. Baseline characteristics

\begin{tabular}{|c|c|c|c|}
\hline & Matched related & Haplo-identical & $\mathrm{p}$-value \\
\hline$N=111$ & 76 & 35 & \\
\hline Age, years (Median/Range) & $18(4-67)$ & $17(8-58)$ & 0.63 \\
\hline Gender (M/F) & $54 / 22$ & $22 / 11$ & 0.65 \\
\hline \multicolumn{4}{|l|}{ Pre-transplant transfusion } \\
\hline$<20$ & 36 & 10 & \\
\hline$>20$ & 40 & 25 & 0.31 \\
\hline Prior IST (N/\%) & $12(15.8 \%)$ & $10(28.5 \%)$ & 0.12 \\
\hline \multicolumn{4}{|l|}{ Risk status（N/\%） } \\
\hline Low & $47(62 \%)$ & $6(17 \%)$ & 0.011 \\
\hline High & $29(38 \%)$ & $29(83 \%)$ & \\
\hline \multicolumn{4}{|l|}{ Stem cell source } \\
\hline PB & 58 & 31 & \\
\hline BM & 16 & 3 & 0.17 \\
\hline $\mathrm{PB}+\mathrm{BM}$ & 2 & 0 & \\
\hline \multicolumn{4}{|l|}{ Conditioning regimen } \\
\hline Flu+Cy+ATG & 57 & 0 & \\
\hline $\mathrm{Flu}+\mathrm{Cy}+\mathrm{TBI}$ & 0 & 26 & 0 \\
\hline $\mathrm{Flu}+\mathrm{Cy}+\mathrm{TBI}+\mathrm{ATG}$ & 0 & 9 & \\
\hline $\mathrm{Flu}+\mathrm{Cy}$ & 19 & 0 & \\
\hline \multicolumn{4}{|l|}{ GVHD prophylaxis } \\
\hline CsA+MTX & 76 & 0 & 0 \\
\hline $\mathrm{PTCY}+\mathrm{Tac}+\mathrm{MMF}$ & 0 & 35 & \\
\hline CD34 cell dose (median) & 5.05 & 7.81 & 0.0004 \\
\hline \multicolumn{4}{|l|}{ Blood group } \\
\hline Identical & 28 & 9 & \\
\hline Major MM & 19 & 12 & \\
\hline Minor MM & 23 & 8 & 0.48 \\
\hline Bidirectional & 6 & 6 & \\
\hline \multicolumn{4}{|l|}{ CMV status } \\
\hline $\mathrm{D}+\mathrm{R}+$ & 69 & 33 & \\
\hline$D+R-$ & 5 & 2 & 0.31 \\
\hline $\mathrm{D}-\mathrm{R}+$ & 2 & 0 & \\
\hline
\end{tabular}

CsA+MTX, cyclosporine and methotrexate; PTCY + Tac +MMF, post-transplant cyclophosphamide, tacrolimus, mycophenolate; $\mathrm{D}$, donor; R, recipient; MM, mismatch.

syndrome/acute myeloid leukemia, and high failure-free survival in matched donor transplantation compared with frontline IST. Furthermore, upfront HID is increasingly being used for patients without a matched donor. Yang et al. compared the outcomes of children and adolescents undergoing IST or upfront HID and showed that upfront HID is superior to $\mathrm{IST}^{4}$. This has led to recent recommendations suggesting that SCT-based treatment should be considered for all patients with SAA over non-SCT, IST-based treatment ${ }^{1}$. The order of preference for donor type should be MRDs, followed by matched unrelated donors and HIDs ${ }^{1}$. We retrospectively analyzed the data of patients who had undergone allo-SCT for SAA (either matched or haplo-identical). The cohort comprised of 111 patients with SAA undergoing allo-SCT (76 patients with MRD, 35 with HID). The majority of our patients were high-risk（total 73/111 [66\%] with 29/76 [38\%] with MRD and 29/35 [83\%] with HID).

$\mathrm{Xu}$ et al. conducted a retrospective registry databasebased study to compare MRD vs. HID for SAA ${ }^{5}$. They found higher grade 2-4 acute GVHD (30.3 vs. 1.5\%, $P<$ 0.001 ) and chronic GVHD (30.6 vs. $4.4 \%, P<0.001)$; however, there was no difference in terms of graft rejection (97.8 vs. $97.1 \%, P=0.528)$, 3-year OS (86.1 vs.
Table 1 B. Outcome analysis of matched vs. haplo-identical transplant patients

\begin{tabular}{|c|c|c|c|}
\hline & Matched & Haplo-identical & $\mathrm{p}$-value \\
\hline$N=111$ & 76 & 35 & \\
\hline \multicolumn{4}{|l|}{ Engraftment $(D+)$} \\
\hline Neutrophil & $12(8-21)$ & $14(12-21)$ & 0.92 \\
\hline Platelet & $18(8-41)$ & $20(9-31)$ & \\
\hline \multicolumn{4}{|l|}{ Mucositis } \\
\hline Gr $1-2(N / \%)$ & $26(34 \%)$ & $13(37 \%)$ & 0.76 \\
\hline Gr $3-4(N / \%)$ & $5(6 \%)$ & $3(8 \%)$ & 0.71 \\
\hline CMV reactivation $(\mathrm{N} / \%)$ & $13(17 \%)$ & $4(11 \%)$ & 0.44 \\
\hline Veno-occlusive disease ( $\mathrm{N} / \%)$ & $2(3 \%)$ & 0 & 0.33 \\
\hline Hemorrhagic cystitis ( $\mathrm{N} / \%)$ & $6(8 \%)$ & $3(8 \%)$ & 0.91 \\
\hline \multicolumn{4}{|l|}{ Acute GVHD } \\
\hline Grade $1(\mathrm{~N} / \%)$ & $9(12 \%)$ & $4(11 \%)$ & 0.95 \\
\hline Grade $2-4 \quad(N / \%)$ & $7(9 \%)$ & $3(9 \%)$ & 0.91 \\
\hline Chronic GVHD（N/\%） & $16(21 \%)$ & $2(6 \%)$ & 0.041 \\
\hline Graft rejection (N/\%) & $4(5 \%)$ & $6(17 \%)$ & 0.042 \\
\hline \multicolumn{4}{|l|}{ Cause of death $(\mathrm{N} / \%)$} \\
\hline Sepsis & $11(14 \%)$ & $19(54 \%)$ & 0.00001 \\
\hline Graft failure & $4(5 \%)$ & - & 0.16 \\
\hline GVHD & $3(4 \%)$ & $1(3 \%)$ & 0.77 \\
\hline VOD & $1(1 \%)$ & - & 0.57 \\
\hline Others & $1(1 \%)$ & - & 0.57 \\
\hline 2-year overall survival & $79 \%$ & $42 \%$ & 0.0001 \\
\hline 2-year disease free survival & $81 \%$ & $32 \%$ & $<0.0001$ \\
\hline
\end{tabular}

$\mathrm{D}+$, number of days post-transplant; Gr, grade of mucositis; VOD, veno-occlusive disease.

$91.3 \%, P=0.358)$, and 3-year failure-free survival $(85.0$ vs. $89.8 \%, P=0.413$ ), justifying the use of upfront HID for patients with SAA lacking a matched donor.

Transplant protocols used for allo-SCT are variable per institutional policy. For MRD SCT, many centers prefer fludarabine and cyclophosphamide, with or without ATG as a conditioning regimen, with cyclosporine and methotrexate as GVHD prophylaxis ${ }^{2,3}$. Generally, the 5-year survival for patients undergoing MRD SCT is age-dependent, being $82 \%$ for those aged $<20$ years, $72 \%$ for those aged 20-40 years, and 53\% for those aged $>40$ years. Primary graft rejection is observed in approximately $20 \%$ patients, with acute and chronic GVHD rates being higher in patients $>40$ years of age $(27 \%)$ than in those $<20$ years of age $(11 \%)^{6}$. For HID SCT, most data from Chinese centers involve the use of a non-PTCYbased protocol ${ }^{7}$, whereas limited data are available on the use of a PTCY-based protocol. Three centers have reported their experience with the use of a PTCY-based platform for HID in $\mathrm{SAA}^{8}$. Generally, graft rejection rates have varied from $0 \%$ to $25 \%$, with acute GVHD rates of approximately $12-13 \%$ and chronic GVHD rates of $0-20 \%$. The 1 -year OS reported is $67-100 \%{ }^{8}$.

We encountered higher graft rejection among the cohort of patients undergoing HID transplantation than among the cohort of patients undergoing MRD transplantation (17\% vs. 5\%). There was no difference between MRD and HID in terms of grade 2-4 acute GVHD (9\% vs. $9 \%, P=0.91)$; however, chronic GVHD was significantly more common in patients undergoing HID transplantation $(21 \%$ vs. $6 \%, P=0.021)$, possibly indicating 

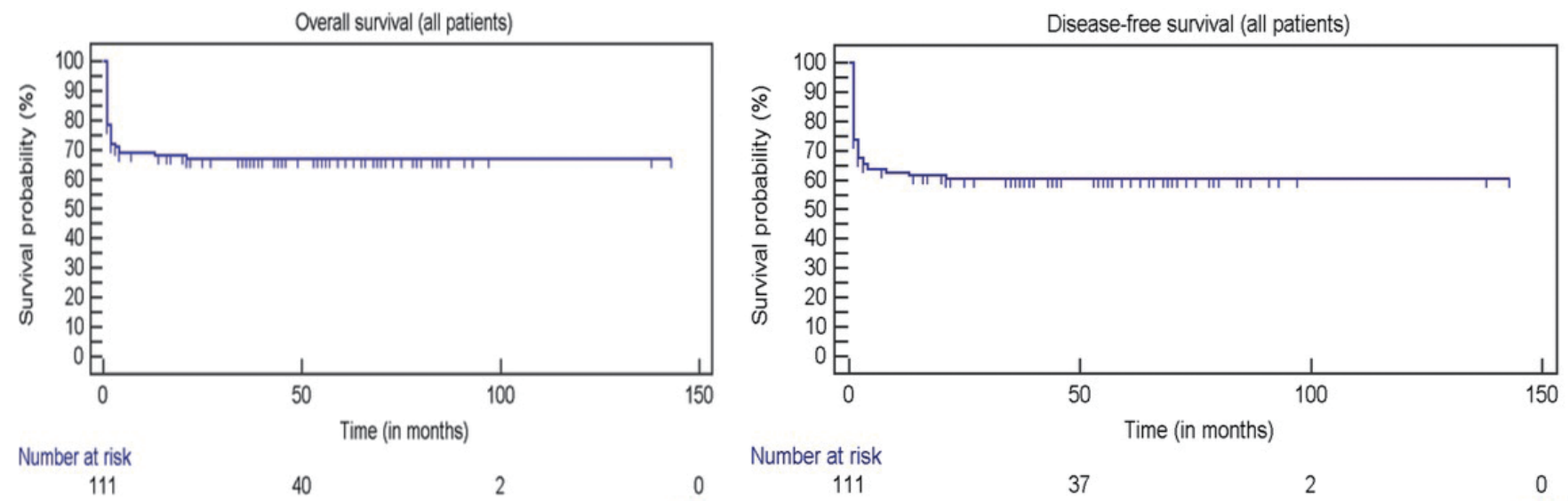

Number at risk

111

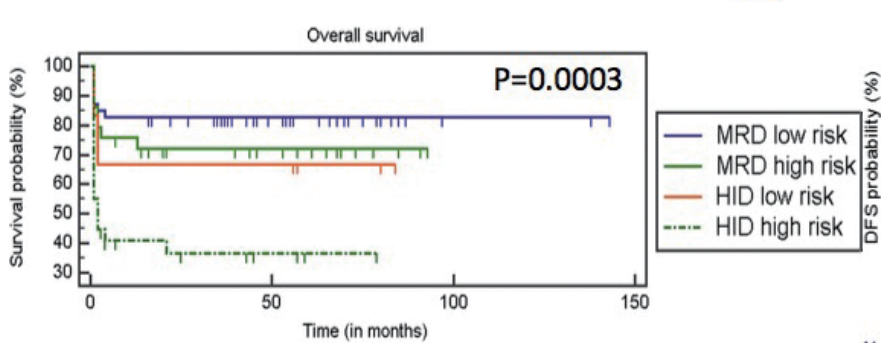

Number at risk

Group: MRD low risk

47
Group: MRD high risk

Group: MRD high risk
29
Group: HID low risk
6

Group: HID high risk

29
40

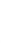

20

12

4

4
2

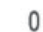

111

37

Figure 1. Kaplan-Meier curves showing overall survival for all patients (1A), and patients stratified according to transplant type and risk status (1C), and disease-free survival for all patients (1B), and patients stratified according to transplant type and risk status (1D)

$1 \mathrm{~A}$ and $1 \mathrm{~B}$ are overall and disease-free survival (all patients). 1C and 1D represent overall and disease-free survival of patients according to donor type and risk status.

MRD, matched sibling donor transplant; HID, haplo-identical donor transplant.

that PTCY is a good agent for chronic GVHD prophylaxis.

Biju et al. analyzed patients with SAA undergoing matched HSCT with fludarabine-based conditioning ${ }^{2}$. They found that high-risk disease status (ATG failure, septic at the time of transplantation, transfusion-dependent) was associated with significantly worse outcomes than low-risk disease status $(64 \%$ vs. $95 \%, P=0.004)$, with a major cause of mortality in the high-risk group being sepsis-related early deaths. In the present study, 58 of 111 patients $(52.2 \%)$ were high-risk, with significantly more high-risk patients in the HID group than in the MRD group ( $38 \%$ for MRD vs. $83 \%$ for HID, $P=0.011$ ). The 2-year OS among all patients was $67 \%$, which was significantly better for MRD transplants and low-risk HID transplants than for high-risk HID transplants $(72 \%$ vs. $32 \%, P=0.0003)$. Similarly, the DFS was significantly better for MRD transplants and low-risk HID transplants than for high-risk HID transplants (71\% vs. $29 \%, P=0.0003)$. The main cause of mortality overall was early death related to sepsis, with high rates of early D +30 mortality (13/35 patients with HID transplants
[37\%] compared with 4/76 patients with MRD transplants $[5.2 \%])$.

We conclude that MRD transplantation is the preferred choice for all transplant-eligible patients. Furthermore, low-risk HID transplantation using PTCY is associated with comparable outcomes to those of MRD transplants in real-world settings; however, sepsis-related mortality in high-risk HID transplantation is of concern. Newer strategies to control sepsis-related transplant morbidity and mortality are urgently needed. Strategies to reduce the risk of graft failure, like using ATG in HID conditioning protocols, may also be considered, although this needs further validation.

\section{Acknowledgements}

We thank Ms. Bharti, Ms. Anubha, and Ms. Saheli who helped in compiling and analyzing the data for the manuscript. 


\section{Author's Contribution}

DC/VK/DD performed transplants, TD/MK/SKS designed research, MK/SKS/RS/AH wrote the manuscript.

\section{Conflict of Interest}

The authors declare no conflict of interest. Disclosure forms provided by the authors are available here.

\section{References}

1. Georges GE, Doney K, Storb R. Severe aplastic anemia: allogeneic bone marrow transplantation as first-line treatment. Blood Adv. 2018; 2: 2020-8.

2. George B, Mathews V, Lakshmi KM, Melinkeri S, Sharma A, Viswabandya A, et al. The use of a fludarabine-based conditioning regimen in patients with severe aplastic anemia-a retrospective analysis from three Indian centers. Clin Transplant. 2013; 27: 923-9.

3. Luznik L, O'Donnell PV, Symons HJ, Chen AR, Leffell MS, Zahurak M, et al. HLA-haploidentical bone marrow transplantation for hematologic malignancies using non-myeloablative conditioning and high-dose post-transplantation cyclophosphamide. Biol Blood Mar Trans. 2008; 14: 641-50.
4. Yang S, Yuan X, Ma R, Jiang L, Guo J, Zang Y, et al. Comparison of Outcomes of Frontline Immunosuppressive Therapy and Frontline Haploidentical Hematopoietic Stem Cell Transplantation for Children with Severe Aplastic Anemia Who Lack an HLA-Matched Sibling Donor. Biol Blood Marrow Transplant. 2019; 25: 975-80.

5. Xu LP, Jin S, Wang SQ, Xia LH, Bai H, Gao SJ, et al. Upfront haploidentical transplant for acquired severe aplastic anemia: registry-based comparison with matched related transplant. Journal of Hematology \& Oncology. 2017; 10: 25.

6. Gupta V, Eapen M, Brazauskas R, Carreras J, Aljurf M, Gale RP, Hale GA, et al. Impact of age on outcomes after bone marrow transplantation for acquired aplastic anemia using HLAmatched sibling donors. Haematologica. 2010; 95: 2119-25

7. Xu LP, Wang SQ, Wu DP, Wang JM, Gao SJ, Jiang M, et al. Haplo-identical transplantation for acquired severe aplastic anaemia in a multicentre prospective study. Br J Haematol. 2016; 175: 265-74.

8. DeZern AE, Brodsky RA. Haploidentical Donor Bone Marrow Transplantation for Severe Aplastic Anemia. Hematol Oncol Clin North Am. 2018; 32: 629-42.

https://doi.org/10.31547/bct-2019-018 Copyright (C) 2020 APBMT. All Rights Reserved. 